\title{
Fratura mandibular associado à remoção de terceiro molar inferior: revisão de literatura
}

\author{
Mandibular fracture associated with the removal of third molar: \\ literature review \\ Fractura mandibular asociada a extracción de tercero molar mandibular: \\ revisión de literatura \\ Valthierre Nunes de Lima \\ Cássio Messias Beija Flor Figueiredo \\ Gustavo Antônio Correa Momesso \\ Sormani Bento Fernandes de Queiros \\ Leonardo Perez Faverani \\ Departamento de Cirurgia e Clínica Integrada, Faculdade de Odontologia, \\ UNESP Univ. Estadual Paulista, 14801-903 Araçatuba - SP, Brasil
}

\begin{abstract}
Resumo
A remoção dos terceiros molares inferiores é um procedimento frequente nas clínicas e consultórios odontológicos. Para remoção destes elementos o profissional deve conhecer os possíveis acidentes ou complicações. A fratura mandibular durante ou após este procedimento é uma complicação que pode ser evitada com o auxílio de exames de imagens adequados e técnica cirúrgica bem executada. Os procedimentos cirúrgicos realizados para remoção destes dentes muitas vezes requerem osteotomias e odontosecção com brocas cirúrgicas e o uso de alavancas, sendo que o mal uso de ambas, associado ao padrão de inclusão geram fragilidade do osso mandibular aumentando a possibilidade de fratura no trans ou pós-operatório. O objetivo deste trabalho foi revisar a literatura sobre os aspectos clínicos e tratamentos realizados para restauração das fraturas mandibulares durante ou após a remoção dos terceiros molares inferiores. Concluímos que a fratura mandibular durante ou após a extração dos terceiros molares inferiores, embora rara, é uma complicação complexa que pode ocorrer durante a aplicação de forças excessivas para remoção do elemento dental ou no pós-operatório durante a mastigação, principalmente nas três primeiras semanas.
\end{abstract}

Descritores: Cirurgia Bucal; Fraturas Mandibulares; Terapêutica.

\begin{abstract}
The removal of third molars is a common procedure in clinics and dental offices. For removal of these elements the surgeon must know the possible accidents or complications. The mandibular fracture during or after this procedure is a complication which should be avoided with the aid of appropriate imaging and surgical technique performed well. The surgical procedures performed to remove these teeth often require osteotomies and odontosection with surgical drills and the use of levers. However, the misuse of both, associated with the inclusion pattern, generate fragility of the mandibular bone increasing the possibility of fracture in the trans or post -operation. The aim of this study was to review the literature on the clinical aspects and treatment provided for restoration of mandibular fractures during or after removal of third molars. We conclude that the mandibular fracture during or after extraction of lower third molars, although rare, is a complex complication that can occur during the application of excessive force to remove the dental element or postoperatively during mastication, especially the first three weeks.
\end{abstract}

Descriptors: Surgery, Oral; Mandibular Fractures; Therapeutics.

\section{Resumen}

La extracción de los terceros molares es un procedimiento común en las clínicas y consultorios dentales. Para la eliminación de estos elementos técnicos deben conocer los posibles accidentes o complicaciones. La fractura mandibular durante o después de este procedimiento es una complicación que debe evitarse con la ayuda de formación de imágenes adecuadas y técnica quirúrgica realizada también. Los procedimientos quirúrgicos para la eliminación de estos dientes a menudo requieren osteotomías y odontosección con fresas quirúrgicas y el uso de palancas, siendo que Sin embargo, el mal uso de ambos, asociado con la inclusión estándar generan fragilidad del hueso de la mandíbula creciente posibilidad de fractura en trans o post-operatorio. El objetivo de este estudio fue revisar la literatura sobre los aspectos clínicos y el tratamiento previsto para la restauración de las fracturas mandibulares durante o después de la extracción de los terceros molares. Llegamos a la conclusión que la fractura mandibular durante o después de la eliminación de los terceros molares, aunque rara, es una complicación complejo que puede ocurrir durante la aplicación de una fuerza excesiva para retirar el elemento dental o después de la operación durante la masticación, especialmente las primeras tres semanas.

Descriptores: Cirurgía Bucal; Fracturas Mandibulares; Terapéutica.

\section{INTRODUÇÃO}

Os terceiros molares inferiores são os últimos dentes a erupcionar, contudo este processo depende de alguns fatores, entre eles o desenvolvimento mandibular. Durante o processo de germinação, o posicionamento destes elementos interfere diretamente no padrão de erupção, podendo apresentar-se, ao final da formação, inclusos ou impactados ${ }^{1}$.

A localização destes dentes fora da arcada pode desenvolver algumas alterações patológicas, devido o contato íntimo com outros elementos dentários, dificuldade de higienização e presença do capuz pericoronário. Tais alterações levam a indicação da remoção destes elementos, a menos que haja alguma contraindicação ${ }^{2}$.

Os procedimentos cirúrgicos realizados para remoção dos terceiros molares inferiores muitas vezes requerem osteotomias e odontosecção com brocas cirúrgicas e o uso de alavancas. Quando da realização inadequada das osteotomias e uso de força excessiva nas alavancas, associados ao padrão de inclusão do terceiro molar, fragilidade pode ser gerada no osso mandibular, 
aumentando a possibilidade de fratura no trans ou pósoperatório ${ }^{3}$.

O osso mandibular apresenta maior resistência em relação ao maxilar e tem como função suportar as forças mastigatórias oclusais, além de resistir todos os músculos da mastigação que se inserem nele. Contudo, apresenta biomecânica complexa e possui articulações do tipo biginglimoartrodial, ou seja, forças exercidas em um lado podem afetar contralateralmente ${ }^{4}$. Considerando a importância deste osso para o sistema estomatognático fraturas que acometam esta região devem ser reparadas o mais rápido possível ${ }^{5}$.

Após a fratura mandibular, o paciente apresenta sinais e sintomas que impossibilitam a correta mastigação e geralmente evolui com quadro infeccioso, se não tratado de forma adequada. $\mathrm{O}$ tratamento destas fraturas muitas vezes necessita de internação hospitalar com anestesia geral e acessos cirúrgicos intra ou extrabucais ${ }^{5}$.

Considerando esta possível complicação, o objetivo deste trabalho foi revisar a literatura sobre os aspectos clínicos e tratamentos realizados para restauração das fraturas mandibulares durante ou após a remoção dos terceiros molares inferiores.

\section{REVISÃO DE LITERATURA}

\section{- Considerações Anatômicas}

A mandibula é o único osso móvel do esqueleto facial e localiza-se na porção inferior do viscerocrânio. Juntamente com o osso hióideo, forma o arcabouço da fixação dos músculos do assoalho bucal. Apresenta um corpo com dois ramos posteriores com trajeto ascendente que na sua porção final encontram-se os côndilos e processos coronóides bilateralmente ${ }^{4}$.

Funcionalmente, a mandíbula suporta e transmite as forças mastigatórias oclusais ao crânio através da articulação temporomandibular (ATM), sem o auxílio de outros ossos. Assim ela possui corticais ósseas bastante espessas e um trabeculado esponjoso que distribui adequadamente as forças que incidem nela ${ }^{6}$.

Ainda em relação À biomecânica mandibular, apresenta zonas de resistência que constituem a trajetória de forças, sendo as principais, a basilar e as alveolares reforçadas anteriormente pelo mento. Os movimentos que transmitem estas forças são realizados fundamentalmente por dois grupos musculares: os supra-hióideos, que atuam abaixando a região anterior, e os músculos da mastigação que atuam no ramo elevando a mandíbula, gerando uma zona de compressão na área basilar e de tensão na região alveolar ${ }^{7}$, como apresentado na Figura $1^{8}$.

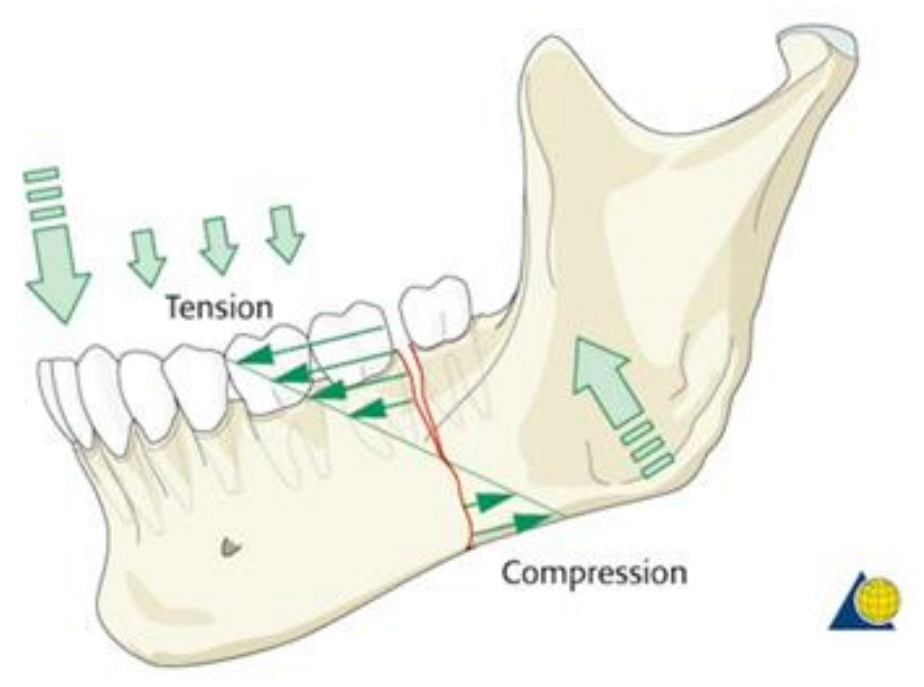

Figura 1: Vetores das forças musculares (Fonte: AOCMF Trauma) ${ }^{8}$.

\section{- Remoção dos terceiros molares inferiores}

Os terceiros molares inferiores (3MI) quando se encontram semi-inclusos, inclusos ou impactados podem gerar alterações patológicas com variados graus de complexidade, por isso indica-se a remoção destes elementos, a menos que haja uma contraindicação ${ }^{9}$. Dessa forma, a remoção dos $3 \mathrm{MI}$ tem como objetivo a prevenção da saúde bucal, evitando a instalação de doença periodontal, cárie, pericoronarite, cistos e tumores odontogénicos, reabsorção radicular e fratura de mandibula ${ }^{10,11}$

Os benefícios advindos da remoção preventiva são superados pela elevada morbidade que o ato cirúrgico representa, devido a contraindicações relativas. Dentre elas, a idade do paciente, a condição médica comprometida e a proximidade ou contato com estruturas nobres ${ }^{12}$.

Pacientes que se encontram em extremos de idade apresentam contraindicações distintas. A falta de colaboração no atendimento de pacientes mais jovens aumenta a dificuldade de visualização e consequentemente as possibilidades de lesões das estruturas nobres ${ }^{13}$. Já os pacientes com idade avançada aumentam a dificuldade da exodontia devido ao decréscimo do turnover ósseo, que gera maior concentração de compacto, bem como menor capacidade de reparação tecidual e consequentemente recuperação do indivíduo ${ }^{14}$.

Há uma diminuição das possibilidades de problemas associados aos dentes inclusos após o final da força de erupção, pois o elemento dentário tende a se fixar em uma posição. Dessa forma, o acompanhamento clínicoradiográfico estará indicado se essa posição for estável, e se no futuro surgir algum problema, pode se indicar a remoção do elemento ${ }^{15}$.

Em indivíduos sistemicamente comprometidos não se indica a remoção preventiva. Em casos de aspecto clínico e/ou radiográfico que demonstre necessidade da remoção, a condição sistêmica deve ser bem avaliada e se possível controlada antes. Em casos de urgência a mesma deverá ser conduzida a nível hospitalar ${ }^{12,16}$.

Estruturas nobres como o nervo alveolar inferior, nervo mentoniano, seio maxilar, fossa nasal, fossa pterigomaxilar, dentre outras podem apresentar intima relação com a posição do elemento incluso, aumentando os riscos de lesões a tais estruturas. Dessa forma, o risco deverá ser avaliado para definição de conduta, pois em alguns casos o acompanhamento poderá ser o melhor tratamento ${ }^{6}$

Lesão do Nervo Alveolar Inferior (NAI) é uma das complicações mais relatadas na literatura, devido sua relação com os 3MI. Um estudo realizado por Blaeser BF, et al. ${ }^{17}$ demostrou que alterações das imagens do canal do NAI e das raízes dos 3MI quando estão em intima relação, o que indica proximidade entre as estruturas e consequentemente maiores possibilidades de lesões.

- Fratura mandibular associada à remoção dos terceiros molares inferiores

A fratura mandibular é uma complicação rara que pode ocorrer durante ou após a remoção dos 3MI. Com a finalidade de minimizar esta possível complicação o profissional deve avaliar o volume dentário, espessura da mandibula e a idade do paciente, pois geralmente este acidente ocorre em pacientes com idade avançada e mandíbulas atróficas ${ }^{18}$.

A aplicação de forças excessivas, geralmente com o uso de alavancas, resulta em fratura mandibular durante a tentativa de remoção dos 3MI. Entretanto, se o dente está profundamente incluso a mandibula encontra-se 
relativamente mais frágil, podendo fraturar com a aplicação de pequena força ${ }^{12}$.

A osteotomia e a odontosecção são etapas fundamentais para remoção destes dentes, seja nos casos de semi-inclusão, inclusão ou impacção. Tal procedimento consiste na remoção de osso ao redor da coroa dentária, com o auxílio de instrumentos rotatórios e brocas cirúrgicas, com a finalidade de exposição da mesma. A posterior odontossecção é realizada para facilitar a trajetória de saída do dente de seu alvéolo e evitar osteotomias extensas que levam a fragilidade mandibular ${ }^{19}$.

Após a remoção de dentes que necessitaram de grandes osteotomias ou inclusões profundas, a mandibula se apresentará atrófica no pós-operatório e cuidados devem ser tomados para evitar a fratura espontânea ${ }^{20}$. O paciente deverá ser informado desta possível complicação e orientado a realizar dieta liquida e pastosa por um período de 4 semanas, tempo suficiente para a reparação óssea e consequente fortalecimento mandibular para receber as cargas mastigatórias normais ${ }^{22}$.

Caso ocorra a fratura no trans ou pós-operatório, exames de imagens são imprescindíveis para o correto diagnóstico e definição de conduta. A radiografia panorâmica é a tomada mais indicada para visualização de fraturas mandibulares, porem a tomografia computadorizada apresenta maiores detalhes sobre a relação da fratura com as possíveis estruturas nobres envolvidas como o NAI (Figura 2$)^{8}$.

Mesmo com todos os cuidados realizados para evitar a fratura mandibular durante ou após a remoção dos terceiros molares inferiores, por se tratar de um acidente, ou seja, algo que não estava planejado, o cirurgião deverá saber lidar com o ocorrido. Se acontecer durante o procedimento, o cirurgião e sua equipe deverão tratar imediatamente, seja por um bloqueio maxilo-mandibular ou por instalação de placas e parafusos de titânio (Figura 3$)^{8}$. Caso ocorra no pós-operatório o paciente deverá contatar imediatamente o profissional para realizar o tratamento o mais rápido possível $^{22}$.

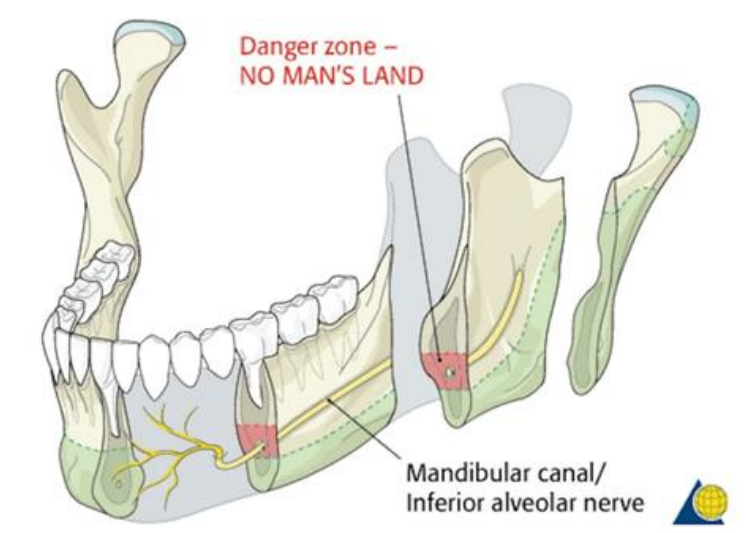

Figura 2: Localização do Nervo Alveolar Inferior (Fonte: AOCMF Trauma) ${ }^{8}$
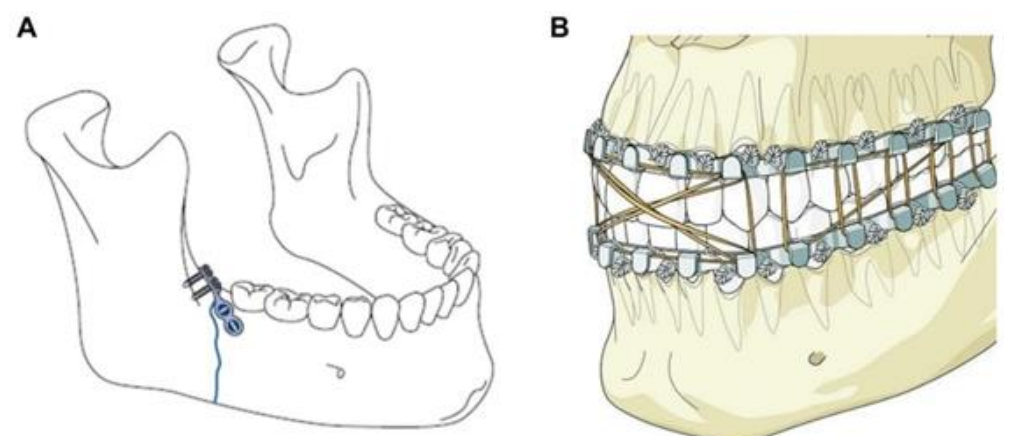

Figura 3: A) Fixação de fratura mandibular com placa e parafusos na zona de tensão; B) Bloqueio maxilomandibular (Fonte: AOCMF Trauma) ${ }^{8}$.
- Tratamento de fratura mandibular após a remoção dos terceiros molares inferiores

$\mathrm{O}$ tratamento das fraturas mandibulares tem como objetivo o restabelecimento oclusal, com reabilitação funcional e estética favorável ${ }^{7}$.

Nos casos de pacientes dentados o questionamento sobre a mudança em sua oclusão após a remoção do dente é importante para o diagnóstico de fratura, pois após o rompimento da base mandibular as forças musculares ganham amplitude para o deslocamento dos cotos, o que gera importantes alterações na oclusão. Em pacientes desdentados, por sua vez, crepitação óssea e mobilidade aparecem como a sintomatologia mais relatada ${ }^{22 .}$

A redução das fraturas pode ser realizada de forma aberta ou fechada. A oclusão, quando possível, é usada como um guia direto para realização de um procedimento adequado. As fraturas podem ser tratadas por fixações internas ou externas (Tabela 1) onde tratamentos conservadores com técnicas de fixação externa são possíveis. Entretanto, na maioria das vezes, fazem-se necessárias as fixações internas? ${ }^{7}$.

Tabela 1. Tipos de fixação

\begin{tabular}{c|c}
\hline Fixação Externa & Fixação Interna \\
\hline Barra em arco com FMM* & Fio de aço \\
Arco de Risdon com FMM* & Parafusos compressivos \\
Anéis de Ivy & Miniplacas \\
Ligadura de Ernest com $\mathrm{FMM}^{*}$ & $\begin{array}{c}\text { Placas de reconstrução } \\
\text { Placas de reconstrução com } \\
\text { Fixador externo }\end{array}$ \\
\hline
\end{tabular}

*FMM-Fixação Maxilo-Mandibular

Um conceito importante na fixação interna das fraturas é o da osteossíntese de carga compartilhada, conhecido como load sharing, e o de carga suportada, conhecido como load bearing (Figura 4$)^{8}$. No conceito do tipo load sharing a adaptação interfragmentária permite alguma justaposição da carga funcional através da fratura fazendo com que a fixação semirígida apropriadamente colocada, resista aos movimentos através da fratura permitindo a cicatrização óssea. Já no conceito load bearing existe pouca ou nenhuma justaposição possível através da fratura, ou seja, o dispositivo fornece fixação suficientemente rígida, prevenido os movimentos e fornecendo um meio adequado para que a cicatrização da fratura ocorra ${ }^{23}$.

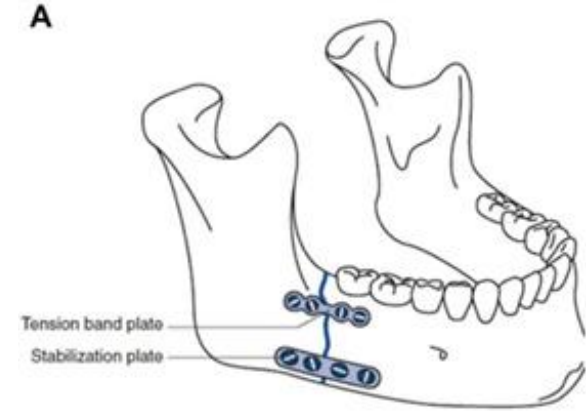

B

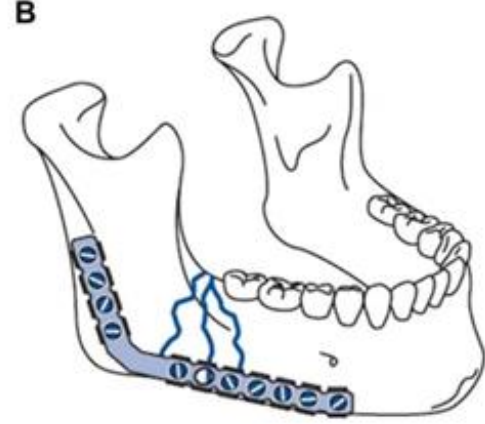

Figura 4: A) Fixação tipo load sharing; B) Fixação tipo load bearing (Fonte: AOCMF Trauma) ${ }^{8}$.

\section{CONSIDERACÕES FINAIS}

Considerando a literatura pesquisada concluímos que a fratura mandibular, embora rara nesses casos, é uma importante complicação que pode ocorrer durante a execussão de forças excessivas para remoção do elemento dental ou mesmo no pós-operatório, durante a mastigação, principalmente nas três primeiras semanas. Para evitar tais 
complicações, o cirurgião deve conhecer os fatores de riscos que podem levar a ocorrência desta complicação e orientar o paciente sobre este possível acidente no pré-operatório, reforçando essas recomendações após o procedimento.

\section{REFERÊNCIAS}

1. Marchiori DF, Packota GV, Boughner JC. Third-molar mineralization as a function of available retromolar space. Acta Odontol Scand. 2016; 74(7):509-17.

2. Ghaeminia H, Perry J, Nienhuijs ME, Toedtling V, Tummers M, Hoppenreijs TJ et al. Surgical removal versus retention for the management of asymptomatic disease-free impacted wisdom teeth. Cochrane Database Syst Rev. 2016(8):CD003879

3. Bodner L, Brennan PA, McLeod NM. Characteristics of iatrogenic mandibular fractures associated with tooth removal: review and analysis of 189 cases. Br J Oral Maxillofac Surg. 2011; 49(7):567-72.

4. Madeira MC. Anatomia da face: bases anatomofuncionais para a prática odontológica. 6. ed. São Paulo: Sarvier; 2008

5. Fonseca R, Barber HD, Powers M, Frost DE. Oral and maxillofacial trauma. 4 th. Saunders; 2013.

6. Reher P, Teixeira LMdS, Reher VGS. Anatomia aplicada à Odontologia. 2. ed. Rio de Janeiro: Guanabara Koogan; 2008

7. Bagheri SC, Bell RB, Khan HA. Terapias atuais em cirurgia bucomaxilofacial. Rio de Janeiro: Elsevier; 2013.

8. AOCMF Trauma. AO Foundation. AO Surgery Reference. Disponível em: https://www2.aofoundation. org $/ \mathrm{wps} /$ portal/surgery? showPage $=$ diagnosis \&bone $=\mathrm{C}$ MF\&segment=Overview\&showCMF=true.

9. Rafetto LK. Managing Impacted Third Molars. Oral Maxillofac Surg Clin North Am. 2015; 27(3):363-71

10. Dodson TB, Susarla SM. Impacted wisdom teeth. BMJ Clin Evid. 2014; pii:1302

11. Bouloux GF, Steed MB, Perciaccante VJ. Complications of third molar surgery. Oral Maxillofac Surg Clin North Am. 2007; 19(1):117-28, vii.

12. Chrcanovic BR, Custodio AL. Considerations of mandibular angle fractures during and after surgery for removal of third molars: a review of the literature. Oral Maxillofac Surg. 2010; 14(2):71-80

13. Kumar PS, Dhupar V, Akkara F, Kumar GB. Eruption status of third molar and its possible influence on the location of mandibular angle fracture: a retrospective analysis. J Maxillofac Oral Surg. 2015; 14(2):243-6

14. Libersa P, Roze D, Cachart T, Libersa JC. Immediate and late mandibular fractures after third molar removal. J Oral Maxillofac Surg. 2002; 60(2):163-5; discussion $5-6$

15. Huang GJ, Cunha-Cruz J, Rothen M, Spiekerman C, Drangsholt M, Anderson L, et al. A prospective study of clinical outcomes related to third molar removal or retention. Am J Public Health. 2014; 104(4):728-34

16. Correa AP, Faverani LP, Ramalho-Ferreira G, Ferreira S, Avila Souza F, de Oliveira Puttini I et al. Unerupted lower third molar extractions and their risks for mandibular fracture. J Craniofac Surg. 2014; 25(3):e228-9.

17. Blaeser BF, August MA, Donoff RB, Kaban LB, Dodson TB. Panoramic radiographic risk factors for inferior alveolar nerve injury after third molar extraction. J Oral Maxillofac Surg. 2003; 61(4):417-21.
18. Ethunandan M, Shanahan D, Patel M. Iatrogenic mandibular fractures following removal of impacted third molars: an analysis of 130 cases. Br Dent J. 2012; 212(4):179-84

19. Wagner KW, Otten JE, Schoen R, Schmelzeisen R. Pathological mandibular fractures following third molar removal. Int J Oral Maxillofac Surg. 2005; 34(7):722-6.

20. Pires WR, Bonardi JP, Faverani LP, Momesso GA, Muñoz XM, Silva AF et al. Late mandibular fracture occurring in the postoperative period after third molar removal: systematic review and analysis of 124 cases. Int J Oral Maxillofac Surg. 2017; 46(1):46-53.

21. Xu JJ, Teng L, Jin XL, Lu JJ, Zhang C. Iatrogenic mandibular fracture associated with third molar removal after mandibular angle osteotectomy. J Craniofac Surg. 2014; 25(3):e263-5.

22. Cankaya AB, Erdem MA, Cakarer S, Cifter M, Oral CK. Iatrogenic mandibular fracture associated with third molar removal. Int J Med Sci. 2011; 8(7):547-53.

23. Miloro M. Princípios de Cirurgia Bucomaxilofacial de Peterson. 2. Ed. São Paulo: Santos; 2008.

\section{CONFLITO DE INTERESSES}

Os autores declaram não haver conflitos de interesse.

\section{AUTOR PARA CORRESPONDÊNCIA}

Cássio Messias Beija Flor Figueiredo

cbcassio04@gmail.com

Submetido em 08/07/2017 Aceito em 10/08/2017 